\title{
Acurácia do Linfonodo Sentinela em Pacientes com Câncer Inicial da Mama Tratadas com Quimioterapia Neoadjuvante
}

\author{
Sentinel Lymph Node Accuracy in Early Breast Cancer Treated with \\ Neoadjuvant Chemotherapy
}

\begin{abstract}
José Roberto Morales Piato, Alfredo Carlos Simões Dornellas de Barros, Katia Maciel Pincerato,
\end{abstract} Adriano Oliveira Vigário e José Aristodemo Pinotti.

\begin{abstract}
RESUMO
Objetivo: avaliar a capacidade preditiva do estudo do linfonodo sentinela (LS) em relação ao estado linfonodal axilar em pacientes com carcinoma invasor inicial de mama submetidas ou não a quimioterapia neoadjuvante.

Métodos: foi realizado estudo prospectivo de 112 pacientes, que foram divididas em dois grupos. O primeiro grupo foi constituido por 70 pacientes que não receberam quimioterapia prévia (Grupo I) e o segundo foi formado por 42 pacientes que foram submetidas a quimioterapia neoadjuvante, com três ciclos do esquema AC (adriamicina + ciclofosfamida) (Grupo II). A resposta à quimioterapia foi parcial $>50 \%$ em 21 pacientes, sendo que em três foi completa, e parcial <50\% em 19 pacientes; em duas pacientes houve progressão da doença. Procedeu-se à injeção peritumoral de dextran marcado com ${ }^{99 m} \mathrm{Tc}$. A mesma foi realizada com auxílio de estereotaxia em 29 pacientes com tumores não palpáveis, sendo 16 do grupo I e 13 do grupo II. Os acúmulos radioativos demonstrados por cintilografia orientaram a biópsia do LS axilar, com auxílio de probe portátil. O estudo anatomopatológico dos LS compreendeu inicialmente um único corte histológico. Os LS livres foram submetidos a cortes seriados a cada $50 \mu \mathrm{m}$ e corados por HE.

Resultados: o LS foi identificado em 108 pacientes. Em três pacientes do grupo I e em uma do grupo II, nas quais não se obteve identificação do LS, as lesões não eram palpáveis. A acurácia do método na predição do estado linfonodal axilar foi de 100\% no grupo de pacientes que não receberam quimioterapia neoadjuvante e de $93 \%$ naquele em que esta forma de tratamento prévio foi utilizada. Essa diferença se mostrou estatisticamente significante.

Conclusões: o presente trabalho possibilitou-nos concluir que em todas as pacientes que não receberam tratamento quimioterápico prévio o estudo do LS foi eficaz para predizer o estado dos linfonodos da axila. A taxa de resultados falso-negativos no grupo de pacientes submetidas à quimioterapia neoadjuvante parece invalidar o emprego do estudo do LS nas mesmas.
\end{abstract}

PALAVRAS-CHAVE: Mama: câncer. Câncer inicial de mama. Linfonodo sentinela. Quimioterapia neoadjuvante. Quimioterapia.

\section{Introdução}

Tendo em vista que o estado linfonodal axilar constitui o mais importante fator prognóstico

Faculdade de Medicina da Universidade de São Paulo - São Paulo USP

Correspondência:

José Roberto Morales Piato

R. Rio de Janeiro, 67, apto. 172

01240-010 - São Paulo - SP

Tel. (11) 3661-3462; TeleFax. (11) 3826-3123

e-mail: piato@uol.com.br isolado do câncer de mama, a dissecção completa da axila tem sido realizada de maneira sistemática. A percepção do baixo índice de comprometimento dos linfonodos da axila nas pacientes com câncer invasivo inicial ${ }^{1}$ mostra, contudo, que essa conduta constitui tratamento excessivo para grande parte das pacientes. Acresce que o esvaziamento axilar completo é responsável por significativa morbidade, especialmente o linfedema de braço. 
Com o intuito de evitar a dissecção axilar rotineira, vários procedimentos foram testados ao longo do tempo, com a finalidade de estabelecer $a$ priori as condições dos linfonodos da axila, especialmente recursos de imagens ${ }^{2,3}$ e análise de certos aspectos anatomopatológicos do tumor primário ${ }^{4}$. Todos esses métodos destinados à predição do estado axilar, entretanto, apresentaram acurácia insatisfatória.

Importantes ensaios clínicos realizados nos últimos anos têm mostrado que o estudo do linfonodo sentinela (LS), seja por técnica de corante ou de isótopos radioativos, parece constituir procedimento altamente promissor para predizer o comprometimento linfonodal axilar, com taxas de acurácia que se situam acima de 95\% ${ }^{5-8}$. Estes elevados índices de acurácia referidos na literatura, assim como aquele obtido no presente ensaio, no grupo de pacientes que não receberam quimioterapia prévia, levam à interessante perspectiva de se preservar a axila em considerável parcela das pacientes com carcinoma mamário em estágio inicial de desenvolvimento.

Questão que permanece em aberto em relação ao método diz respeito à sua utilização em pacientes submetidas a quimioterapia neoadjuvante. Por admitir a possibilidade de que o achado de linfonodos livres após tratamento quimioterápico decorra ou de ausência de metástases ou de esterilização pela terapêutica, Veronesi et al. ${ }^{9}$ são de opinião que o conhecimento do estado linfonodal axilar pelo estudo do LS seja obtido antes da instituição de tratamento quimioterápico primário.

A propósito deste tema, em recente trabalho, que envolveu 38 pacientes com câncer mamário nos estádios II e III submetidas a quimioterapia neoadjuvante, Cohen et al. ${ }^{10}$ analisaram a acurácia do estudo do LS para a predição do estado axilar. Os resultados obtidos por estes autores mostraram indices de resultados falso-negativos significativamente maiores, quando comparados com aqueles observados em outros estudos feitos em pacientes que não receberam qualquer tratamento prévio.

O presente trabalho teve como objetivo verificar a acurácia do estudo do linfonodo sentinela na predição do estado linfonodal axilar em pacientes sem qualquer tratamento prévio e naquelas submetidas a quimioterapia neoadjuvante.

\section{Pacientes e Métodos}

Para a realização do presente ensaio foram selecionadas 112 mulheres portadoras de carcinoma invasor de mama, com tumores primários
T1 e T2 e axilas clinicamente livres. A idade das pacientes variou de 26 a 85 anos, com média de 53,2 anos e mediana de 51,5 anos.

Foram excluídas do estudo gestantes, lactantes, portadoras de tumores multicêntricos e pacientes previamente submetidas a qualquer tipo de cirurgia na mama ou na axila, inclusive biópsias incisional ou excisional.

As pacientes foram divididas em dois grupos, assinalando-se que não houve diferença estatisticamente significante no que se refere aos diâmetros do tumor primário entre os mesmos. O critério de mensuração do tumor pela ultrasonografia foi a medida do maior diâmetro. No Grupo I, que foi constituido por 70 pacientes, sendo 54 com tumor palpável e 16 com lesão não palpável, não se utilizou tratamento quimioterápico prévio, ao passo que no Grupo II, que foi formado por 42 pacientes, sendo 29 com tumor palpável e $13 \mathrm{com}$ lesão não palpável, empregou-se quimioterapia neoadjuvante.

O estudo do LS nas pacientes do Grupo II foi precedido da administração de três ciclos do esquema $\mathrm{AC}$ (adriamicina: $60 \mathrm{mg} / \mathrm{m}^{2}$ e ciclofosfamida: $600 \mathrm{mg} / \mathrm{m}^{2}$ ), com intervalos de 21 dias. Mediante exames ultra-sonográficos das mamas destas pacientes observamos que em 21 ocorreu resposta parcial com redução maior que 50\% das dimensões do tumor, sendo que em três foi completa; resposta parcial com redução menor que $50 \%$ foi observada em 19 pacientes e em duas houve progressão da doença.

No dia anterior à cirurgia procedeu-se à injeção peritumoral de 0,2 mL de dextran 70, marcado pelo ${ }^{99 \mathrm{~m}} \mathrm{Tc}$ com atividade de $15 \mathrm{MBq}$. O colóide utilizado apresenta partículas com diâmetro menor que $50 \mathrm{~nm}$, consideradas de pequena dimensão. Nos casos de tumor não palpável, por ocasião do diagnóstico ou após emprego da quimioterapia, a injeção foi realizada com auxílio de estereotaxia, seguindo a técnica ROLL (radioguided occult lesion localization) ${ }^{11}$. Ressalte-se que nestas pacientes o emprego do referido colóide possibilitou a localização simultânea da lesão primária e do LS.

A localização pré-operatória do LS axilar foi obtida por meio de linfocintilografia, utilizando-se gama câmara convencional. No momento da cirurgia empregou-se detector de radiações gama (sonda); a radiação captada por este aparelho, traduzida em sinais acústicos, orientou a biópsia do LS. Considerou-se como sendo LS tanto aquele linfonodo de maior contagem como os que eventualmente apresentaram contagem de no minimo $10 \%$ do mesmo.

Nos casos em que se praticou cirurgia conservadora (quadrantectomia), a biópsia do LS foi, sempre que possivel, realizada por meio da mes- 
ma incisão cirúrgica, após a retirada do sítio de injeção do radiofármaco. Esta medida mostrou-se bastante eficaz para evitar a interferência decorrente da proximidade dos sítios radioativos, em especial nos casos de tumores localizados em quadrantes laterais. Em seguida foi realizada a dissecção axilar completa, para estabelecer correlação anatomopatológica do LS com os demais linfonodos.

Com a finalidade de avaliar a proteção radiológica foram feitas medidas de taxa de exposição durante 20 procedimentos consecutivos, com auxílio de dois detectores do tipo Geiger-Miller. A medida realizada a $0,5 \mathrm{~cm}$ do sítio de injeção revelou que a radiação absorvida foi de 0,05 + 0,01 $\mathrm{mSv}$ e de $0,003+0,0009 \mathrm{mSv}$ a $100 \mathrm{~cm}$ da paciente.

Os linfonodos identificados pela sonda foram submetidos a estudo intra-operatório, utilizandose a técnica de imprint das superficies de corte dos mesmos, praticadas a cada $3 \mathrm{~mm}$. Com vistas a se evitar perda de material, não se realizou exame histopatológico pela técnica de congelação.

Em seguida, a patologista efetuou exame histopatológico definitivo do LS e dos demais linfonodos da axila. O LS foi inteiramente incluído em parafina, sendo que um corte de cada bloco, com espessura de $4 \mu \mathrm{m}$, corado por $\mathrm{HE}$ foi selecionado para exame. Os demais linfonodos axilares foram submetidos à mesma técnica de exame.

Nos casos em que foram identificadas células neoplásicas no LS, encerrou-se o estudo anatomopatológico. Nos demais casos realizaram-se cortes seriados a cada $50 \mu \mathrm{m}$, com espessura igualmente de $4 \mu \mathrm{m}$, obtendo-se, desta forma, cinco a seis lâminas adicionais coradas por HE.

Para caracterizar a população estudada procedemos à análise descritiva dos dados, observando as distribuições de freqüência para as variáveis qualitativas e calculando as médias, medianas e desvios padrão mínimo e máximo, para as variáveis quantitativas. O nível de significância nos testes foi de $5 \%(\alpha)$.

\section{Resultados}

A identificação do LS na axila foi possivel em 108 pacientes $(96,4 \%)$. Nas quatro pacientes em que não se observou captação do radiofármaco (sendo três delas do Grupo I e uma do Grupo II), os tumores primários eram não palpáveis. Em 106 pacientes o LS foi identificado no nivel I da axila e nas seis restantes no nivel II. Em relação a este aspecto, não se observou diferença significativa entre os dois grupos estudados.

No que se refere à acurácia para a predição do estado axilar, a taxa foi de $100 \%$ no grupo de pacientes sem quimioterapia prévia, uma vez que não foram observados resultados falso-negativos, isto é, LS livre e metástases em outros linfonodos da axila (Tabela 1).

Tabela 1 - Estado do linfonodo sentinela (LS) e dos demais linfonodos da axila para o grupo sem quimioterapia neoadjuvante (grupo I).

\begin{tabular}{lcc}
\hline Estado do LS & Axila livre & Axila comprometida \\
\hline Livre & $52(100 \%)$ & 0 \\
Comprometido & 0 & $18(100 \%)$ \\
Total & $52(100 \%)$ & $18(100 \%)$ \\
\hline
\end{tabular}

Em três pacientes que receberam quimioterapia neoadjuvante, o estudo anatomopatológico definitivo do LS, por meio de cortes seriados, mostrou ausência de metástases, ao passo que aquele realizado nos demais linfonodos da axila evidenciou comprometimento por células neoplásicas. Desta forma, observou-se indice de resultados falso-negativos de $17 \%$; a taxa de acurácia do método para a predição do estado linfonodal axilar foi, portanto, de 93\% (Tabela 2).

Tabela 2 - Estado do linfonodo sentinela (LS) e dos demais linfonodos da axila no grupo submetido a quimioterapia neoadjuvante (grupo II).

\begin{tabular}{lcc}
\hline Estado do LS & Axila livre & Axila comprometida \\
\hline Livre & $23(100 \%)$ & $3(16,7 \%)$ \\
Comprometido & 0 & $15(83,3 \%)$ \\
Total & $23(100 \%)$ & $18(100 \%)$ \\
\hline
\end{tabular}

\section{Discussão}

Pela análise da literatura observam-se opiniões amplamente favoráveis ao emprego de dissecção axilar seletiva guiada pelo estudo do LS em câncer inicial de mama. Conclusões definitivas, contudo, só poderão ser obtidas quando os diversos ensaios clínicos estiverem em andamento, comparando o tratamento padrão com aquele baseado na cirurgia axilar radioguiada e demonstrando a mesma evolução em termos de sobrevida ${ }^{12}$.

Em revisões bibliográficas observa-se nitida predileção pelo emprego da técnica de isotopos radioativos para o estudo do $\mathrm{LS}$, uma vez que a mesma tem sido considerada a mais eficiente, além de ser rápida, simples, sensivel e desacompanhada de morbidade. Estudo realizado por Veronesi et al. ${ }^{9}$ comprovou que não existem 
riscos biológicos para a equipe cirúrgica ligados à exposição às radiações. Pudemos confirmar tal segurança por meio do emprego de detectores do tipo Geiger-Miller em 20 procedimentos consecutivos.

Independentemente do local da injeção do radiofármaco, isto é, peritumoral ou na pele de revestimento do tumor ou da região para-areolar do quadrante de localização do tumor, ocorre marcação do $\mathrm{LS}^{13}$. Esta constatação parece evidenciar que o LS constitui não apenas o primeiro linfonodo a receber a drenagem linfática do tumor, mas também aquele principal de toda a rede linfática da mama.

$\mathrm{Na}$ literatura observa-se que a remoção cirúrgica do LS orientada pela sonda é feita no início da cirurgia, através de incisão separada de 2$4 \mathrm{~cm}$, praticada na região axilar ${ }^{9,14,15}$. Esta técnica cirúrgica pode ocasionar dificuldade na diferenciação entre a radiação proveniente do sitio de injeção e aquela emitida pelos linfonodos marcados, especialmente quando o tumor primário situa-se em quadrantes laterais. Por tal motivo, nossa opção foi pela remoção inicialmente do sítio da injeção e, posteriormente, dos linfonodos marcados pelo radiofármaco. Estes procedimentos foram, sempre que possivel, realizados pela mesma incisão cirúrgica.

Aspecto que se reveste de grande importância é o aprimoramento do estudo anatomopatológico do LS, com vistas a se evitarem resultados falso-negativos. Tendo em vista questionamentos acerca da possibilidade de passarem despercebidas micrometástases por ocasião do exame do LS, é interessante analisar métodos mais sensiveis de avaliação anatomopatológica de linfonodos e tecer considerações críticas acerca dos mesmos.

Weaver et al. ${ }^{16}$ verificaram que a técnica de imuno-histoquímica apresenta grande sensibilidade na detecção de micrometástase em linfonodos, uma vez que apresenta capacidade para identificar células cancerosas isoladas ou pequenos agregados das mesmas localizados em parênquima, cápsula ou sinusóide dos mesmos. Cabe ressaltar, contudo, que esta técnica tem o inconveniente de propiciar resultados falso-positivos em parte significativa dos casos, uma vez que células epiteliais não neoplásicas, assim como células névicas eventualmente presentes em linfonodos, podem ser confundidas com células neoplásicas ${ }^{17}$.

Um método não morfológico ainda mais refinado para a identificação de metástases em linfonodo é a reação em cadeia da polimerase (PCR), uma vez que apresenta altíssima sensibilidade, com potencial para detectar uma única célula neoplásica em um milhão de células ${ }^{18}$. Esta téc- nica igualmente tem o sério inconveniente de apresentar resultados falso-positivos, quando presentes no linfonodo inclusões epiteliais benignas ou células leucêmicas.

No presente estudo optamos pelo exame anatomopatológico do LS por meio de cortes seriados do mesmo a cada $50 \mu \mathrm{m}$; esta decisão baseouse em importante ensaio de Nasser et al. ${ }^{19}$. Em estudo de 159 casos, em que utilizaram a referida técnica para o exame anatomopatológico de linfonodos axilares de portadoras de câncer de mama, estes autores conseguiram identificar micrometástases com dimensões iguais ou superiores a $0,2 \mathrm{~mm}$. Desta forma, puderam verificar que o método apresenta a mesma eficácia que a técnica de imuno-histoquímica para a identificação de metástases com essas dimensões.

A utilização de cortes seriados do LS demonstrou ser procedimento bastante eficaz no nosso estudo, uma vez que reduziu o índice de resultados falso-negativos de quatro casos $(25 \%)$ para três casos $(17 \%)$ no grupo de pacientes submetidas a quimioterapia neoadjuvante. Em relação ao grupo sem tratamento quimioterápico, este método ocasionou a detecção de micrometástases maiores que $0,2 \mu \mathrm{m}$ no LS, que não haviam sido diagnosticadas pelo exame convencional. Neste grupo, o método acrescentou casos de axila comprometida a expensas unicamente do LS.

Resta discutir o problema das eventuais micrometástases com dimensões inferiores a 0,2 $\mu \mathrm{m}$, assunto que também foi exaustivamente analisado por Nasser et al. ${ }^{19}$. Pelo ensaio prospectivo de 50 pacientes com metástases em linfonodos axilares menores que $0,2 \mu \mathrm{m}$ ou ainda aquelas detectadas apenas por imuno-histoquimica, estes autores verificaram que a taxa de sobrevida das mesmas foi semelhante à de pacientes com linfonodos negativos.

Tal comportamento biológico das micrometástases linfonodais com menos de 0,2 $\mu \mathrm{m}$ talvez possa ser explicado por hipótese recentemente aventada por $\operatorname{Rosser}^{20}$. Do ponto de vista deste autor, tais micrometástases não representariam um passo na história natural do câncer mamário. Decorreriam, possivelmente, de traumas ocasionados na mama pelos procedimentos empregados na identificação e na remoção do LS, especialmente as massagens que se efetuam após a injeção do radiofármaco.

Conforme assinalado na introdução deste artigo, as taxas de acurácia para predição do estado linfonodal axilar obtidas por vários autores, em estudos que envolveram grande número de pacientes não submetidas a quimioterapia neoadjuvante, foram superiores a 95\%, sem atingir, con- 
tudo, a totalidade dos $\operatorname{casos}^{5-9}$. No presente traba1ho, que se relaciona com número não tão elevado de pacientes estudadas, observamos indice de acurácia de $100 \%$. É de se esperar que, com o progressivo aumento da casuística, surjam resultados falso-negativos, a exemplo do observado em grandes séries.

O estudo que ora apresentamos revelou que a acurácia do estudo do LS para a predição do estado axilar no grupo de pacientes submetidas a quimioterapia neoadjuvante foi insatisfatória. Em suas pacientes tratadas previamente com quimioterápicos, Cohen et al. ${ }^{10}$ igualmente tiveram taxa de resultados falso-negativos relativamente elevada, isto é, de $17 \%$.

Ainda não dispomos de dados para explicar de forma definitiva as causas que levam a quimioterapia neoadjuvante a ocasionar prejuízo da acurácia na predição do estado axilar. Com vistas a obter algum esclarecimento, procuramos analisar o comportamento dos linfonodos axilares comprometidos por metástases sob a ação de agentes antiblásticos.

Uma primeira hipótese estaria relacionada com esterilização de linfonodos comprometidos. Em estudo randomizado de 1.523 portadoras de tumores primários T1, T2 e T3 e axilas N0 e N1, Fisher et al. ${ }^{21}$ verificaram que aquelas tratadas pela associação de doxorrubicina e ciclofosfamida apresentaram indice de axilas positivas de 43\%, ao passo que as do grupo que não recebeu tratamento quimioterápico exibiram taxa de 59\%. Em nosso estudo, entretanto, não verificamos diferenças significativas em termos de comprometimento dos linfonodos axilares, quando comparadas as pacientes dos dois grupos.

Outra hipótese estaria relacionada com alterações morfológicas dos linfonodos. Em ensaio que envolveu 89 mulheres afetadas por câncer de mama submetidas a quimioterapia neoadjuvante com esquema de doxorrubicina e ciclofosfamida, Sharkey et al. ${ }^{22}$ constataram que em nove ocorreu fibrose focal hialina similar àquela encontrada na regressão do tumor primário, sendo que em cinco havia fibrose associada a tumor metastático residual e nos demais apenas fibrose. No presente ensaio, contudo, não observamos diferença quanto à presença de metástases em ambos os grupos. Além disso, o estudo histopatológico realizado nos LS dos três casos falso-negativos não revelou a presença de fibrose focal hialina.

Em termos práticos, os resultados do presente trabalho validam a metodologia do estudo do LS com emprego de isótopos radioativos. Parece ser ainda cedo para uma delimitação precisa de sua aplicação fora dos protocolos clínicos controlados em instituições especializadas. Em certas condições, com lesões iniciais e circunscritas, havendo disponibilidade tecnológica e tendo a equipe cirúrgica ultrapassado a fase de aprendizado, o oferecimento da metodologia do LS já pode fazer parte da rotina de atendimento.

Até que se possa dispor de dados esclarecedores acerca da interferência da quimioterapia neoadjuvante na acurácia do estudo do LS na predição do estado linfonodal axilar em mulheres com câncer inicial de mama, é recomendável que o método seja utilizado somente em pacientes sem tratamento quimioterápico prévio.

\section{ABSTRACT}

Purpose: to evaluate the predictive capacity of the sentinel lymph node (SLN) in relation to the axillary lymph node status in patients with initial invasive breast carcinoma submitted or not to neoadjuvant chemotherapy.

Method: a prospective study was performed in 112 patients divided into two groups. The first group comprised 70 patients who had not received previous chemotherapy (Group I) and the second consisted of 42 patients who were submitted to neoadjuvant chemotherapy in three cycles of AC (adriamycin + cyclophosphamide) (Group II). Regarding chemotherapy, we observed partial response $>50 \%$ in 21 patients, being complete in three of them, and $<50 \%$ in 19 patients; in two patients progression of the disease occurred. A peritumoral injection of ${ }^{99 m}$ Tc dextran was applied with the help of stereotaxy in 29 patients with nonpalpable tumors, 16 of Group I and 13 of Group II. The radioactive accumulation shown by scintigraphy guided the biopsy of the axillary SLN with the help of a probe. The anatomopathologic study of SLN was based initially on a single section. When the LSN was free, it was submitted to serial sections at $50 \mu$ m intervals, stained with $H E$.

Results: SLN was identified in 108 patients. No identification has been obtained in four patients, all with nonpalpable lesions (3 patients of Group I and 1 of Group II). The method's accuracy in predicting the axillary lymph node status was $100 \%$ in patients who did not receive neoadjuvant chemotherapy and $93 \%$ in those to whom this kind of treatment was administered. This difference proved to be statistically significant.

Conclusions: the present study allowed us to conclude that in all patients who did not receive previous chemotherapy treatment, the SLN study was effective in predicting the axillary lymph node status. The high rate of false-negative results in the group of patients submitted to neoadjuvant chemotherapy seems to invalidate the use of SLN study these patients.

KEY WORDS: Breast cancer. Sentinel lymph node. Neoadjuvant chemotherapy. 


\section{Referências}

1. Silverstein MJ, Gierson ED, Waisman JR, Senofsky GM, Colburn WJ, Gamagami P. Axillary lymph node dissection for T1a breast carcinoma. Is it indicated? Cancer 1994; 73:664-7.

2. Pinotti JA. Estudo da linfografia na propedêutica das metástases linfáticas do carcinoma de mama [tese]. Campinas: Universidade Estadual de Campinas. Faculdade de Ciências Médicas; 1970.

3. Venta L. Evaluation of axillary node status with imaging modalities. Semin Breast Dis 1998; $1: 134-40$.

4. Barth A, Craig PH, Silverstein MJ. Predictors of axillary lymph node metastases in patients with T1 breast carcinoma. Cancer 1997; 79:1918-22.

5. Veronesi U, Paganelli G, Galimberti V, et al. Sentinelnode biopsy to avoid axillary dissection in breast cancer with clinically negative lymph-nodes. Lancet 1997 ; 349:1864-7.

6. Giuliano AE, Jones RC, Brennan M, Statman R. Sentinel lymphadenectomy in breast cancer. J Clin Oncol 1997; 15:2345-50.

7. Borgstein PJ, Pijpers R, Comans EF, Van Diest PJ, Boom RP, Meijer S. Sentinel lymph node biopsy in breast cancer: guidelines and pitfalls of lymphoscintigraphy and gamma probe detection. J Am Coll Surg 1998; 186:275-83.

8. Krag DN, Ashikaga T, Harlow SP, Weaver DL. Development of sentinel node targeting technique in breast cancer patients. Breast $\mathrm{J}$ 1998; 4:67-74.

9. Veronesi U, Paganelli G, Viale G, et al. Sentinel lymph node biopsy and axillary dissection in breast cancer: results in a large series. J Natl Cancer Inst 1999; 91:368-73.

10.Cohen LF, Breslin TM, Kuerer HM, Ross MI, Hunt KK, Sahin AA. Identification and evaluation of axillary sentinel lymph nodes in patients with breast carcinoma treated with neoadjuvant chemotherapy. Am J Surg Pathol 2000; 24:126672 .

11.Zurrida S, Galimberti V, Monti S, Luini A. Radioguided localization of occult breast lesions. Breast 1998; 7:11-3.
12. Barros ACSD. Linfonodo sentinela no câncer de mama: tarde para ignorar, cedo para concluir. Rev Bras Mastol 2000; 10:53-4.

13.Roumen RM, Geuskens LM, Valkenburg JG. In search of the true sentinel node by different injection techniques in breast cancer patients. Eur J Surg Oncol 1999; 25:347-51.

14.Albertini JJ, Lyman GH, Cox C, et al. Lymphatic mapping and sentinel node biopsy in the patient with breast cancer. JAMA 1996; 276:1818-22.

15.Feldman SM, Krag DN, McNally RK, Moor BB, Weaver DL, Klein P. Limitation in gamma probe localization on the sentinel node in breast cancer patients with large excisional biopsy. J Am Coll Surg 1999; 188:248-54.

16. Weaver DL, Krag DN, Ashikaga T, Harlow SP, O'Connell M. Pathologic analysis of sentinel and nonsentinel lymph nodes in breast carcinoma: a multicenter study. Cancer 2000; 88:1099-107.

17.Fisher ER, Swamidoss S, Lee CH, Rockette H, Redmond C, Fisher B. Detection and significance of occult axillary node metastases in patients with invasive breast cancer. Cancer 1978; 42:2025-31.

18.Ghossein RA, Rosai J. Polymerase chain reaction in the detection of micrometastases and circulating tumor cells. Cancer 1996; 78:10-6.

19.Nasser IA, Lee AK, Bosari S, Saganich R, Heatley G, Silverman ML. Occult axillary lymph node metastases in "node-negative" breast carcinoma. Hum Pathol 1993; 24:950-7.

20.Rosser RJ. A point of view: trauma is the cause of occult micrometastatic breast cancer in sentinel axillary lymph nodes. Breast J 2000; 6:209-12.

21.Fisher B, Brown A, Mamounas E, et al. Effect of preoperative chemotherapy on local-regional disease in women with operable breast cancer: findings from National Surgical Adjuvant Breast and Bowel project B-18. J Clin Oncol 1997; 15:2483-93.

22. Sharkey FE, Addington SL, Fowler LJ, Page CP, Cruz AB. Effects of preoperative chemotherapy on the morphology of resectable breast carcinoma. Mod Pathol 1996; 9:893-900. 